\title{
Marcas lingüísticas en la prensa escrita de México como estrategias discursivas para evadir responsabilidad al informar sobre narcotráfico
}

\author{
» Linguistic marks in the written press of Mexico as \\ discursive strategies to evade responsibility when \\ reporting on drug trafficking
}

\author{
Édgar Ávila González - Iván Ávila González \\ Universidad Michoacana de San Nicolás de Hidalqo en Morelia, Michoacán, México
}

\section{Resumen}

La presencia del narcotráfico en Michoacán ha sido foco de atención en la agenda nacional e internacional de la prensa, sin embargo, informar sobre el tema resulta una tarea complicada por la responsabilidad de las voces reproducidas en la enunciación periodística; por lo que los reporteros a través de la práctica han elaborado fórmulas específicas para no comprometerse con la veracidad de lo afirmado en la noticia. Tal es el caso del uso de los adverbios como marcas evidenciales estratégicas para referir a la fuente de información cuando en una construcción periodística se publica sobre la presencia del narcotráfico. La presente investigación que se centra en el análisis de notas informativas extraídas de tres diarios michoacanos también plantea la existencia de otras marcas lingüísticas, además de los adverbios, que pueden cubrir las características de un evidencial como marcador dentro de una enunciación como es el caso de presunto y supuesto.

Palabras clave: narcotráfico, adverbios, evidenciales, enunciación, prensa.

\begin{abstract}
The presence of drug trafficking in Michoacán has been the focus of attention of the national and international media agenda, however, reporting on the issue is a complicated task due to the responsibility of the voices reproduced in the journalistic enunciation; so the reporters through the practice have developed specific formulas to not commit to the truth of what is stated in the news. Such is the case of the use of adverbs as strategic evidential marks to refer to the source of information when a journalistic construction is published on the presence of drug trafficking. The present investigation that focuses on the analysis of informative notes extracted from three Michoacán newspapers also raises the existence of other linguistic marks, in addition to adverbs, that can cover the characteristics of an evidential as a marker within an enunciation such as the case of presumed and supposed.
\end{abstract}

Keywords: drug trafficking, adverbs, evidential, enunciation, press.

Cómo citar este artículo:

Ávila González, E. \& Avila González, I. (2019). Marcas lingüisticas en la prensa escrita de México como estrategias discursivas para evadir responsabilidad al informar sobre narcotráfico. Revista Amauta, 17333), pp.167-178. http://dx.doi.org/10.15648/am.33.2019.11

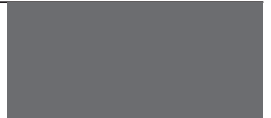

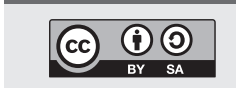

() 2019

Correspondencia de autor:

Iccedgar.avila@gmail.com afavordelomejor85@ hotmail.com

Recibido:

25/Septiembre/2018

Aceptado:

15/Diciembre/2018

Publicado:

3/Enero/2019 


\section{Introducción}

La multiplicidad de voces inmersas en el mensaje periodístico es un tema que durante varios años ha merecido una amplia atención en los estudios del discurso; esta polifonía la usa como estrategia el redactor de textos para intentar persuadir de la veracidad del contenido que reproduce, y de esta manera, señalar al destinatario la valorización que da a las enunciaciones emitidas por voces ajenas'. A esa reproducción de un discurso presentado como ajeno, en forma de enunciados o pensamientos atribuibles a otro, se le conoce como Discurso Referido (en adelante DR). El DR tiene diversas representaciones discursivas a las que da alojamiento un texto periodístico, como es el Discurso Directo, Discurso Indirecto, Discurso Indirecto Libre, citas mixtas y citas no expresas que bien recapitula Frías (2012) en su artículo Sobre las formas de reproducción del discurso ajeno en algunos textos periodísticos de la prensa italiana y española. Sin embargo, no son las únicas expresiones de la lengua que refieren a una fuente u origen de algún conocimiento, también existen otras marcas discursivas -0 al menos eso hemos propuesto- denominadas evidenciales, que señalan al elemento lingüístico que refiere a la fuente de información (Cornillie, 2016; Torner, 2016 \& Bermúdez, 2005).

Bermúdez (2005) apunta que los periódicos a través de la práctica, han elaborado "fórmulas específicas que aluden al origen de la información impresa" como es el caso de "según dejaron trascender fuentes oficiales" y "fuentes confiables" (p.1). En atención a lo anterior es que decidimos plantear un análisis del uso de los evidenciales en la prensa escrita de Michoacán, cuando se publica sobre la presencia del narcotráfico con especial énfasis en el uso de los adverbios, para observar cómo en los textos periodísticos, propone Torner (2016), esas marcas evidenciales se utilizan como recurso mediante los cuales el reportero o periodista inserta en su enunciado marcas explícitas sobre la fuente de la que ha obtenido la información. Para mostrar tal supuesto -engarzado a nuestro interés indagatorio - hemos trazado la siguiente pregunta de investigación: ¿cuál es el propósito del uso de adverbios como marcadores evidenciales en la prensa escrita de Michoacán? En ese sentido, planteamos tres objetivos: 1) identificar los adverbios como marcadores evidenciales en los

1 Ducrot (1986) plantea la teoría de la polifonía de la enunciación a partir de los estudios de Bajtín desarrollados en el primer cuarto del siglo anterior. En su propuesta teórica explica, entre otras cosas, que son polifónicos los textos periodísticos elaborados por el redactor de un medio de comunicación porque en ellos hay varias voces enunciadoras. 
textos periodísticos sobre la presencia del narcotráfico y 2) analizar el uso de los marcadores evidenciales de los diarios de Michoacán como marcas para no comprometerse con la verdad de lo afirmado en la enunciación reproducida.

Debido a que el acontecimiento noticia es una interpretación construida de una realidad social en la cual una persona -reportero o periodista- está inmersa, el siguiente análisis se realiza desde una perspectiva pragmática, para el cual se han tomado algunos fragmentos de notas informativas de los periódicos La Voz de Michoacán, Cambio de Michoacán y Diario Provincia, correspondientes al periodo de febrero-agosto de 2016, cuando distintos medios informativos publicaron sobre la aparición de mantas en el municipio de Zamora, Michoacán, México. En ellas se leía un mensaje que anunciaba la presencia de organizaciones del narcotráfico, a pesar de que en enero del mismo año las autoridades habían anunciado la desaparición de células delictivas en esa zona geográfica.

\section{El contexto necesario}

Los acontecimientos de inseguridad que México ha vivido en los últimos años como consecuencia de la presencia del narcotráfico, han situado al país en el centro de atención de la agenda mediática nacional e internacional. Este fenómeno social no es un asunto nuevo, desde hace décadas cárteles de la droga han disputado entre ellos y con el Estado mexicano la posición de supremacía como grupo delincuencial de una determinada zona geográfica. Los medios informativos han convertido todo lo referente a estas actividades del narcotráfico en parte sustancial de su cobertura periodística, de tal manera, que se han realizado varios análisis de las estrategias discursivas utilizadas en la construcción textual, a fin de observar cómo el tema es abordado por los productores de información y cómo es interpretado por los destinatarios. Una de las regiones donde se ha focalizado este fenómeno ha sido Michoacán, luego de la caída de los "Zetas" y del "Cártel del Golfo", organizaciones criminales que en 2006 desataron una jornada de violencia y confrontaciones por buscar el predominio del Estado.

De manera específica en el caso de Michoacán, a partir de la década de 1990 surgió el "Cártel del Milenio" o de "los hermanos Valencia". En el periodo de 2001-2005, la entidad tuvo como organización delictiva hegemónica a los "Zetas", y más tarde 
como producto de una escisión surgió "La Familia Michoacana", grupo delincuencial dominante hasta el 2010, año en el que la organización se transformó en "Los Caballeros Templarios". Durante todo ese tiempo, los gobiernos federal y estatal emplearon operativos de seguridad en ese Estado con la intención de disminuir los hechos violentos y de inseguridad. Se realizaron estrategias para capturar a las "cabecillas" del narco, inclusive en 2009, el entonces presidente de la República, Felipe Calderón Hinojosa le declaró la guerra a la delincuencia organizada. Los resultados de estas diligencias en materia de seguridad fueron acontecimientos de interés para los medios.

En la continuación de tácticas políticas y de seguridad para desaparecer a los grupos delincuenciales que se habían apoderado del control de varios sectores de la sociedad en Michoacán, en 2014 el presidente de la República, Enrique Peña Nieto crea la figura del comisionado para la seguridad y el desarrollo integral de Michoacán y al mando instala a Alfredo Castillo Cervantes. La llegada de Castillo tuvo lugar el 13 de enero de ese año cuando el entonces gobernador de Michoacán, Fausto Vallejo Figueroa (2012-2014), solicitó la intervención del Gobierno Federal tras varios sucesos violentos registrados en la entidad por la lucha contra el narcotráfico. El hecho principal que dio paso a que se pidiera ayuda en materia de seguridad fue el incendio provocado, el 10 de enero, al Palacio Municipal de Apatzingán, entre otros inmuebles y tiendas comerciales de la localidad. Según autoridades competentes en materia de seguridad, se atribuyó el hecho a grupos delincuenciales. De esa forma, Vallejo Figueroa aceptó que la inseguridad había rebasado la capacidad del Estado de poder contenerla.

En ese momento, el cártel que se convirtió en objetivo principal del gobierno federal y estatal fue el de los llamados "Caballeros Templarios", una partidura de la célula criminal antes conocida como "La Familia Michoacana". Sin embargo, el gobierno además de dicha situación tenía otro problema: pacificar el conflicto de las "Autodefensas", un movimiento de ciudadanos armados, creado el 13 de febrero de 2013 en el municipio de Tepalcatepec con el argumento de que si el gobierno no daba las necesarias garantías de seguridad, el pueblo se haría cargo de proteger a las familias.

El desarrollo de esta problemática social y la necesidad de los gobiernos de regresar el orden y la tranquilidad fue de interés mediático para la prensa local, nacional e 
incluso internacional. Michoacán era nota diaria por la inseguridad que permeaba en esa época.

\section{Soporte teórico}

De acuerdo con Bermúdez (2005) se llama evidencialidad² al dominio semántico relacionado con la fuente u origen de la información expresada en el enunciado; y evidencial al elemento lingüístico que marca o refiere a la fuente de información. Este autor menciona que las posibilidades referenciales en la enunciación son varias. En principio el hablante puede haber tenido contacto directo (visual o de otro tipo) con la situación descrita, haber tenido contacto no con la situación misma pero sí con indicios que apuntan hacia esa situación, o haber recibido información de una tercera persona. En ese sentido, la evidencia puede ser directa, razonada o transmitida. Destaca que en el español, a diferencia de otras lenguas como el quechua, el tuyuca o el turco, suele clasificarse entre las lenguas que solo poseen marcadores evidenciales léxicos, en otras palabras, que no han gramatizalizado el dominio de evidencialidad, y esto se puede observar en expresiones como "por lo visto", "según dicen", "aparentemente", "según parece", etc.

De acuerdo con Cornillie (2016), el campo funcional de la evidencialidad se expresa mediante adverbios, adjetivos, usos parentéticos de verbos de comunicación, verbos auxiliares y tiempos verbales. De los cuales, como ya hemos mencionado, focalizaremos la atención en el campo funcional de los adverbios, pues son las marcas lingüísticas de interés para el presente trabajo. Bermúdez (2005) y Torner (2016) hacen alusión a adverbios del español como marcadores evidenciales, entre ellos, mencionan los siguientes cinco: aparentemente, evidentemente, obviamente, supuestamente y visiblemente que han sido clasificados como evidenciales en diversos estudios. Torner (2016) hace hincapié en que los mencionados adverbios tienen un significado próximo, indirectamente relacionado con el valor de verdad de la oración y agrega que estos intensifican 0 atenúan la fuerza de lo que se asevera, "se trata, por lo tanto, de modificadores que, al menos en su uso prototípico, son externos al contenido proposicional, pues indicen en el nivel del acto enunciativo"

2 Haciendo eco en las investigaciones de Bermúdez (2005), el término evidencialidad apareció por primera vez en los estudios de la lingüística a través de un trabajo de Boas (1947) sobre la gramática del Kwakiutl. Ello se consolidaría más tarde en los trabajos de Jakobson (1957) y la publicación del volumen Evidentiality: The linguistic coding of epistemology de Chafe y Nichols (1986). 
(p.251). Torner indica que a su vez estos adverbios pueden dividirse ${ }^{3}$ en dos series que corresponden con adverbios relacionados con el valor de verdad de la oración. Estas divisiones obedecen a lo siguiente:

- A. aparentemente, supuestamente

- B. evidentemente, obviamente, visiblemente

Torner (2016) apunta que los adverbios de la serie A son ejemplos de restrictores del valor de verdad y que su función consiste en poner en suspenso el juicio sobre el valor de verdad de la oración. Añade que su función consiste en indicar una suspensión de la evaluación de verdad condicional de la proposición, a pesar de que existan indicios que podrían llevar a considerar la proposición como verdadera. "La función de estos adverbios es, pues, doble: por un lado, indican que hay datos que permitirían suponer que la proposición es cierta; por otro, conllevan una suposición del valor de verdad" (p.3).

Por otro lado, el autor describe que los adverbios de la serie B son reforzadores del valor de verdad. Estos presuponen que la oración es cierta y presentan este valor de verdad como algo incuestionable. Explica que la evaluación sobre el valor de verdad de la oración resulta de un proceso de inferencia a partir de ese conocimiento compartido, "como consecuencia del dicho valor de verdad es presentado como evidente" (Torner, 2016, p.259).

\section{El uso de evidenciales en la prensa escrita de Michoacán}

Tomando como base teórica lo expuesto en el apartado anterior presentamos el primer ejemplo de adverbios como formas evidenciales en la enunciación discursiva. Los estudios de Torner (2016) señalan que supuestamente pertenece a los adverbios restrictores del valor de verdad. Esta marca lingüística indica que la oración a la que modifica describe que la realidad que se expresa es una suposición como podemos observarlo a continuación:

3 Figueras (2014) extiende esa lista de adverbios a ciertamente, evidentemente, obviamente, incuestionablemente, indiscutiblemente, indudablemente, naturalmente, de verdad. De los cuales afirma que constituyen intensificadores (refuerzan el contenido proposicional); mientras que aparentemente, presuntamente, supuestamente, al parecer o por lo visto, establecen atenuadores. 
(1) Luego de los bloqueos carreteros registrados el pasado martes en Los Reyes y Buenavista Tomatlán supuestamente por integrantes de los grupos de autodefensa en protesta por la detención de "El Americano", en donde prendieron fuego a dos automóviles, se le preguntó al mandatario estatal sobre el particular y su respuesta fue que los operativos se pusieron en marcha en aquella región con la intención de detener a toda persona armada que se dedique a cometer actos delictivos, incluso el perredista enfatizó en que la estrategia de seguridad "no se detendrá" en la entidad ni tampoco "habrá vuelta de página" para encerrar a los culpables que han puesto "por delante a niños y mujeres con la intención de delinquir". (Martínez, La Voz de Michoacán, 25/02/2016: 8A)

En el ejemplo (1), el reportero reproduce un hecho que puede asegurar y que además tiene un antecedente, lo podemos ver en Luego y en el pasado martes, donde hace referencia a bloqueos carreteros en dos municipios de Michoacán, pero al integrar supuestamente la continuación de la enunciación pierde la veracidad y se mantiene en una realidad supuesta y no necesariamente cierta. El redactor del texto periodístico, a pesar del antecedente marcado en el inicio del fragmento textual, cancela la responsabilidad a integrantes de grupos de autodefensa. Utiliza el adverbio supuestamente para referir a otras voces que sí lo han dicho, que sí tienen la certeza de lo enunciado, pero en su proposición el redactor del texto periodístico se mantiene en un espacio de no responsabilidad por los resultados interpretativos de lo escrito.

Continuando con la perspectiva teórica de Torner (2016), la función de "presuntamente, pretendidamente o presumiblemente consiste en indicar una suspensión de la evaluación veritativo condicional de la proposición" (p.3), a pesar de que la proposición tenga indicios de que pueda ser considerada como verdadera. Veamos el siguiente ejemplo:

(2) De acuerdo a un comunicado, ayer se llevó a cabo una acción operativa que ha permitido, hasta el momento, la detención de siete personas que tenían en su poder armas de diferentes calibres y gasolina, quienes presuntamente participaron en la obstrucción de 
vías de comunicación en diferentes puntos de la entidad. (Apro, Cambio de Michoacán, 13/04/2016: 7)

En el anterior ejemplo podemos observar que hay elementos que indican que la proposición es verdadera, la enunciación relatada por el redactor de textos periodísticos menciona "la detención de siete sujetos que tenían en su poder armas de diferentes calibres y gasolina", pero al mencionar el pronombre relativo en plural y a continuación, presuntamente, se cancela el valor de verdad, y de esta forma, se suspende la veracidad del seguimiento de la enunciación "participaron en la construcción de vías de comunicación en diferentes puntos de la entidad". El reportero no asume la responsabilidad de redactar la participación de algunas personas en el acontecimiento.

Al igual que (2), en (3) el reportero hace uso del adverbio presuntamente después de argumentar que "Marín se convirtió en uno de los objetivos principales de los gobiernos estatal y federal" para anular la responsabilidad de que este era el líder de una célula criminal. Utiliza la marca lingüística para referir a una fuente de información, es decir, eso infiere que existe un alguien que sí conoce que Marín encabezaba un grupo delictivo, y por ello hace mención de ese dato, pero el reportero no lo quiere constatar. A partir de ahí, el adverbio indica que la correspondencia entre la realidad y lo descrito por una expresión lingüística es supuesta.

(3) Marín se convirtió en uno de los objetivos principales de los gobiernos estatal y federal, incluso en abril de este 2016 la célula criminal que presuntamente encabezaba agredió a tiros a la Policía de Ixtlán, ataque que dejó un oficial lesionado. A raíz de ello se capturó a cuatro supuestos operadores de El Pelón Marín, uno de ellos de su alta confianza: Gerardo V, apodado La Astilla y/o El Flaco. Con la detención de Marín la PGJE da un fuerte golpe a la estructura delictiva de Los Viagras y el compromiso es desmantelar a dicho grupo, afirmaron fuentes al interior de la oficina del procurador. (Red-113, Cambio de Michoacán, 18/06/2016: 25)

Ahora bien, en este trabajo de investigación, también queremos destacar la existencia de otras marcas lingüísticas como supuesto y presunto que pueden cubrir las características de un evidencial como marcador dentro de una enunciación que refiere a 
la fuente de información. Torner (2016) acota que supuestamente hereda su significado de su base derivativa, es decir, el participio del verbo suponer. En este caso como adjetivo puede indicar el resultado de una acción de suponer. Para demostrar esto, retomemos el ejemplo (3). En la proposición donde comienza a leerse "a raíz de ello se capturó a cuatro", el valor de verdad es atribuido, pero al hacer uso de supuestos para dar seguimiento a "operadores de El Pelón Marín", se pierde el valor de verdad. Además, el sustantivo en plural, operadores, engarzado directamente a El Pelón Marín indica solo una suposición de que estos estén relacionados con la persona mencionada con un mote.

Continuando con este planteamiento, en (4) el uso del adjetivo supuestas refiere a una acción de suponer algo, adquiere un valor intencional y se antepone al sustantivo organizaciones, pero se posiciona delante del sustantivo mensajes. Lo cual da como inferencia que, para el reportero, mensajes está en un espacio de realidad, pero, por lo contrario, supone que existan las organizaciones delictivas en la enunciación, y que tal vez los mensajes pertenezcan a otros enunciadores que desconoce el redactor:

(4) Silvano Aureoles Conejo se refirió a las mantas con mensajes de supuestas organizaciones delictivas y que aparecieron en los municipios de Zamora y Lázaro Cárdenas, e indicó que ya fueron detenidos algunos de los responsables y se inició la investigación para dar con sus patrones. (Elizalde, Diario Provincia, 08/02/2016: 7)

En (5), la suposición infiere que el surgimiento de un nuevo grupo delictivo en Michoacán es alejada de la realidad y no hay compromiso del reportero para afirmar una enunciación de esta índole, y por lo tanto, en una primera lectura se percibe que deja al secretario de gobierno con la responsabilidad de la enunciación del "supuesto surgimiento de un grupo delictivo en Michoacán":

(5) Luego de reconocer que "sigue habiendo personas con la intención de usar el discurso social para justificar acciones ilícitas y es no lo podemos permitir", el secretario de Gobierno Adrián López Solís, explicó con respecto al supuesto surgimiento de un nuevo grupo delictivo en Michoacán, que las autoridades tienen la obligación de corroborar la veracidad, el alcance y la dimensión de esta manifestación, pues con la facilidad que tienen las redes sociales se pueden hacer este tipo 
de mensajes y que anuncios de esta naturaleza se pueden confirmar.

(Miranda, La Voz de Michoacán, 09/02/2016: 11A)

De la misma forma, en (6) presuntos indica una posible falta de correspondencia entre lo expresado en la proposición y la realidad. Dice Torner (2016) que no existe un referente de quién está emitiendo el juicio de verdad y por tal motivo se pone en duda si los autores pertenecen a una posible realidad, aunque sí es un saber que se supone compartido. De esta forma el reportero disminuye la fuerza en la enunciación de la detención de los autores, pues al hacer uso de una presunción coloca a los autores en un juicio de valor acerca de si son responsables o no.

(6) Explicó que los presuntos autores de la colocación de las mantas fueron detenidos inmediatamente, ya que el gobierno del estado tiene los elementos necesarios para frenar la delincuencia organizada. (Ávila, Diario Provincia, 11/02/2016: 3)

\section{Conclusiones}

En esta investigación hemos observado que existen unas marcas lingüísticas denominadas adverbios, que inmersas en una enunciación pueden adquirir el carácter de evidencial, cuando estas refieren a la fuente de información, un lugar donde se emitió un conocimiento que en una expresión textual -en este caso las notas informativas- hace eco y es un recurso habitual de los reporteros o periodistas para quitarse responsabilidad de lo dicho. Se trata de una estrategia discursiva para presentar un discurso como ajeno a quien escribe, pues un discurso referido sirve para no responsabilizarse de la interpretación que tendrá lo expuesto en la nota periodística 4 .

En ese sentido podemos responder a nuestra pregunta de investigación, y aventurarnos a realizar la siguiente afirmación, el redactor de textos informativos hace

\footnotetext{
4 El uso de los adverbios y adjetivos como marcas evidenciales en las construcciones periodísticas que se proponen en este trabajo, también podría abrir la discusión para una investigación sobre el posible empleo de estos elementos lingüísticos como estrategia conveniente de la prensa michoacana para adaptarse al Nuevo Sistema de Justicia Penal, y de esta manera, garantizar y privilegiar la presunción de inocencia de la persona enunciada en la construcción textual, que es uno de los diversos objetivos del sistema penal acusatorio que entró en vigor en Michoacán en febrero de 2014. Para más información sobre este tema puede consultarse el artículo de Leñero y Carranza La construcción de la noticia en el sistema penal acusatorio, publicado en 2014.
} 
uso de los evidenciales para no comprometerse con la verdad de lo aseverado en la enunciación reproducida, en este caso, la prensa en Michoacán hace uso de los adverbios como marcas evidenciales para no responsabilizarse de las construcciones textuales sobre la presencia del narcotráfico.

Los evidenciales atienden a fórmulas características de los medios de comunicación impresos que aluden al origen del conocimiento. Para fines de este trabajo se observó que en los textos periodísticos se sugiere -por lo menos en los ejemplos que hemos presentado- a un discurso de los representantes de la autoridad estatal, quienes han manifestado los trabajos en materia de seguridad para resarcir la presencia del narcotráfico.

Durante el desarrollo del presente trabajo vimos que el uso de los adverbios, presuntamente y supuestamente que atenúan la fuerza de lo que se asevera, crean un marco de una realidad que puede ser cancelado. Pudimos observar que en una enunciación expresada estas marcas lingüísticas pueden tener algunos usos básicos como contrastar dos situaciones, es decir, una que se afirma y otra que se suspende, apartarse de la responsabilidad discursiva y reproducir una emisión discursiva ajena.

Por último, hacemos hincapié en el planteamiento expuesto sobre la existencia de otras marcas lingüísticas, además de los adverbios, que pueden cubrir las características de un evidencial como marcador dentro de una enunciación, es el caso de presunto y supuesto. El planteamiento pudiera ser un poco aventurado porque necesitaríamos un marco teórico más amplio y un análisis gramatical de mayor profundidad. Sin embargo, en esta breve investigación intentamos destacar esa característica de evidencial que pudieran tener para referir a la fuente de información o de conocimiento.

Intentamos señalar que estas marcas, en algunos casos, indican una posible falta de correspondencia entre lo expresado en la proposición y la realidad; en concreto, en una nota informativa es nulo el referente sobre quién emite la enunciación discursiva, pero se puede inferir ese quién por el desarrollo que presenta el cuerpo del texto periodístico. 


\section{Referencias bibliográficas}

Apro (13 de abril de 2016). Consignana a 19 viagras. Cambio de Michoacán, p.7.

Ávila, É. (11 de febrero de 2016). Rechazan riesgo de nuevos cárteles. Diario Provincia, p.3.

Bermúdez, F. (2005). Evidencialidad: La codificación lingüística del punto de vista (tesis doctoral). Universidad de Estocolmo, Estocolmo, Suecia.

Cornillie, B. (2016). En prensa. Los auxiliares evidenciales en español. En R.González Ruiz, D. Izquierdo Alegría y Ó. Loureda Lamas (eds.), La evidencialidad en español: teoría y descripción. (pp.227-250). Editorial Vervuert/Iberoamericana.

Ducrot, 0. (1986). El decir y lo dicho, polifonía de la enunciación. Barcelona: Paidós Comunicación.

Elizalde, A. (08 de febrero de 2016). Silvano: No habrá margen para el crimen. Diario Provincia, 7.

Figueras, C. (2014). La relevancia de los adverbios evidenciales. Comunicación presentada en el XI Congreso Internacional de Lingüística General. Universidad de Navarra, 21-23 mayo de 2014. Disponible en https://www.academia.edu/9979353 /La_relevancia_de_los_adverbios_evidenciales

Frías, M. (2012). Sobre las formas de reproducción del discurso ajeno en algunos textos periodísticos de la prensa italiana y española. Philologia Hispalensis, 26(3-4), 121-153.

Leñero, S. y Carranza, E. (2014). La construcción de la noticia en el sistema penal acusatorio. México: Impretei.

Martínez, G. (25 de febrero de 2016). Hay 7 órdenes por bloqueos. La Voz de Michoacán, 8 A.

Miranda, F. (09 de febrero de 2016). Los Viagras no son riesgo en la entidad. La Voz de Michoacán, 11A.

Red-113 (18 de julio de 2016). PGJE da fuerte golpe a Los Viagra, captura a El Pelón Marín. Cambio de Michoacán, p.25.

Torner, S. (2016). En prensa. Los adverbios evidenciales en español. En R. González Ruiz, D. Izquierdo Alegría y Ó. Loureda Lamas (eds.): La evidencialidad en español: teoría y descripción. (pp.251-279). Editorial Vervuert/Iberoamericana. 